\title{
Perceived sexual dysfunction in breast cancer survivors
}

\author{
Terri A Zomerlei ${ }^{1}$, Elizabeth A Block ${ }^{2}$, Jamie L Caughran ${ }^{3,4}$ and Jessica L. Keto ${ }^{3,4 *}$ \\ ${ }^{1}$ Department of Plastic Surgery, The Ohio State University Wexner Medical Center, Columbus, USA \\ ${ }^{2}$ Department of Psychology, University of Michigan, Ann Arbor, USA \\ ${ }^{3}$ Grand Rapids Medical Education Partners in partnership with Michigan State University General Surgery Residency, Grand Rapids, USA \\ ${ }^{4}$ Mercy Health Saint Mary's Comprehensive Breast Center, Grand Rapids, Michigan, USA
}

\begin{abstract}
Purpose: While sexual dysfunction after breast cancer treatment has been documented in the literature, the prevalence, degree of dysfunction, and how best to address this issue in survivors is less clear. The goal of this study was to determine the prevalence of patient perceived sexual dysfunction in breast cancer survivors.

Patients and Methods: Patients were identified using a hospital sponsored cancer registry. Adult female patients with stage 0-III breast cancer diagnosed between 2008 and 2011 were eligible for enrollment. Study participants received a 30 question survey assessing the their demographic and breast cancer treatment information, perceived impact on sexual function due to treatment, overall sexual satisfaction, and factors potentially interfering with sexual function.

Results: Of the 407 women meeting inclusion criteria, 125 returned an informed consent, and 75 completed the survey. Sixty-four percent of responders felt that treatment had a negative impact on their sexual function. Of those, only $23 \%$ discussed the issue with a care provider while $60 \%$ desired a physician initiated conversation. A negative perception was strongly associated with decreased satisfaction with intimate relationships ( $\mathrm{p}<0.001)$.

Conclusions: Patient perceived sexual dysfunction is a well-documented consequence of breast cancer treatment. Our data demonstrate that many patients feel this issue is not addressed during routine care; however, desire a discussion with their treating physician. Sexual dysfunction is independent of age, time from surgery, and interfering factors making it difficult to identify high risk populations. Therefore, sexual dysfunction should be identified in all breast cancer survivors during routine follow-up with interventions made when appropriate.
\end{abstract}

\section{Introduction}

Improved cancer detection and treatment methods have resulted in an increased number of cancer survivors over the past several decades $[1,2]$. Meeting the unique physical and psychological needs of this growing population is a rapidly evolving field in cancer care. A report from the Institute of Medicine has previously highlighted the medical community's deficits in the awareness of cancer survivor care [3]. In response, the creation of high quality survivorship programs was cited as being essential for addressing unmet needs [2]. Survivorship programs have become integral for providing a support network and the appropriate referral services $[3,4]$. Existing literature demonstrates that residual symptom distress and psychological needs that are not addressed by the medical system negatively impact a patient's overall well-being [4].

The occurrence rate of such sexual and interrelationship difficulties has been reported to range from $15 \%$ for reduced physiological arousal to $64 \%$ for reduced sexual desire [5]. Factors that contribute to dysfunction include long-term treatment-related side effects such as pain with intercourse and fatigue. These symptoms are commonly documented as long-term side effects related to breast cancer treatment. Patient perceived sexual dysfunction contributes to an already difficult and stressful time in their life and is not limited to duration of treatment $[5,6]$. While this problem is acknowledged, few data are available documenting the extent of sexual dysfunction in breast cancer survivors and if this dysfunction is being adequately addressed by their medical team.

\section{Patients and Methods}

English speaking, female patients between the ages of 18 and 80 diagnosed with breast cancer stages 0 -III were identified using a hospital sponsored cancer registry. All patients had undergone surgical intervention for breast cancer at a single surgical practice between August 2008 and December of 2011. A total of 407 patients met the inclusion criteria. These women received a study packet by mail containing a cover letter describing the research study which included all elements of informed consent and a separate consent form with a stamped return envelope. One hundred twenty-five women completed a consent form and subsequently received a 30 question survey in the mail. The authors utilized several methods previously demonstrated to improve response to mailed surveys including follow-up contact, university sponsorship, assurance of confidentiality, and first class mail $[7,8]$.

The survey was comprised of 30 multiple-choice questions divided into four sections. The first two portions of the survey contained investigator developed questions related to demographics and selfperceived quality of sexual function after the treatment of breast cancer. The remaining survey questions consisted of two validated

${ }^{\star}$ Correspondence to: Jessica Keto, Director, Comprehensive Breast Center, The Lacks Cancer Center, Mercy Health Saint Mary's, 250 Cherry Street, Grand Rapids, MI 49503, USA, Tel: (616) 685-6756; Fax: (616) 685-8961; E-mail: ketojl@mercyhealth.com

Key words: cancer, oncology, breast, survivorship, sexual function

Received: May 14, 2018; Accepted: June 04, 2018; Published: June 07, 2018 
question banks from the PROMIS ${ }^{\circ}$ tool (Patient Reported Outcomes Measurement Information System); a self-assessment tool developed by the National Institute of Health. The PROMIS question banks were designed to assess patients' overall sexual satisfaction and physical factors potentially interfering with sexual function $[9,10]$.

A total of 90 women returned completed surveys. Survey responses based on a Lickert scale were converted to numerical values and SPSS Statistics, version 21 (IBM, Armonk, NY) was used for the statistical procedures. Data were analyzed using the chi-square test and Spearman's rank correlation coefficient. Significance was assessed at $\mathrm{p}<0.05$.

\section{Results}

\section{Description of survey responders}

In total, 407 women met inclusion criteria and received a letter of explanation and an informed consent by mail. One hundred and twenty five women returned informed consents and subsequently received a survey in the mail. A total of 90 anonymous surveys were returned with 75 surveys being complete $(60 \%$ response rate for those with informed consent, $18 \%$ of the total patient population). Fifteen surveys were returned with incomplete survey portions and were discarded.

Demographic and clinical information of the 75 respondents is summarized in Table 1. The median age range was 51-60 years of age. Median time post-surgery was 2 years (range $<1$ years to 4 years). The majority of the respondents identified with the Caucasian race (98\%) and remaining were African American (2\%). Fifty-six (75\%) of the survey responders were married. A total of 44 (59\%) of women endorsed current use of endocrine therapy or chemotherapy. Twenty-nine (39\%) of women had a lumpectomy while 46 (61\%) had a mastectomy. Thirty-five $(76 \%)$ of the mastectomy group had a subsequent breast reconstruction, either autologous or with implants.

\section{Dysfunction assessment}

To access patient perceived sexual dysfunction, participants graded their level of agreement with the following "impact" statement, "I feel that my cancer treatment had a negative impact on my sexual function." Forty-seven (64\%) of women wither agreed strongly agreed or were neutral with the impact statement. These 47 patients were then asked to answer further questions regarding their discomfort. Twenty-two (47\%) of this group reported having emotional distress as a result of this negative impact. Eleven women (23\%) had discussed this distress with their physician, while 29 (62\%) desired a physicianinitiated conversation. Twenty-three (49\%) had a desire to discuss the impact on sexual functioning with a counselor.

\section{Sexual satisfaction and response to impact statement}

Sexual satisfaction was assessed within our population using the responses to seven questions graded on a Likert scale (strongly agree, agree, neutral, disagree, strongly disagree). Each response was assigned a numerical value and then combined to create a satisfaction score. For example, if a person answered strongly disagreed $(\mathrm{SD}=0)$, to all seven questions, their total score would be 0 , while a response of strongly agree $(\mathrm{SA}=4)$ to all questions would generate a score of 28 . Subsequent analysis revealed a statistically significant $(\mathrm{p}<0.01)$ correlation between a satisfaction score and the response to the impact question. Those with a higher score were more likely to disagree with the impact statement (i.e. felt their cancer treatment had little to no impact on their sexual functioning), while those with a lower score were more likely to have some level of agreement with the impact statement. The relationship between the satisfaction score and the response to the impact statement is shown in Figure 1.

\section{Interfering factors}

Responders were asked to select what, if any, physical factors were interfering with their sexual function. The most commonly cited interfering factors were fatigue (56\%), dyspareunia (35\%), scars (45\%) and hot flashes (45\%) (Table 2). Weight gain, weight loss, hair loss and use of a medical device (such as a chemotherapy port) were cited as interfering factors by less than $5 \%$ of responders. The presence of scars was the only factor that had a positive correlation with agreement to the impact statement and was more frequently cited by the group of women who felt that breast cancer treatment had negatively impacted their sexual function $(\mathrm{p}=0.04)$.

\section{Relationship to surgical procedure}

Of the 29 women that had undergone a lumpectomy, $42 \%$ perceived a negative impact on their sexual functioning. In contrast,

Table 1. Survey responder demographic and clinical information.

\begin{tabular}{|l|l|}
\hline Variable & Value \\
\hline Age & \multicolumn{2}{l|}{} \\
\hline $31-40$ years & $4 / 75(5.3 \%)$ \\
\hline $41-50$ years & $16 / 75(21.3 \%)$ \\
\hline $51-60$ years & $30 / 75(40.0 \%)$ \\
\hline $61-70$ years & $15 / 75(20 \%)$ \\
\hline $71-80$ years & $10 / 75(13.3 \%)$ \\
\hline Race (\% Caucasian) & $72 / 75(96.0 \%)$ \\
\hline Relationship Status $(\%$ Married/long term relationship) & $64 / 75(85.3 \%)$ \\
\hline Time post surgery & \multicolumn{2}{|l|}{} \\
\hline$<6$ months & $1 / 75(1.3 \%)$ \\
\hline 6 to 12 months & $8 / 75(10.7 \%)$ \\
\hline$>12$ to 18 months & $16 / 75(21.3 \%)$ \\
\hline$>18$ to 24 months & $11 / 75(14.7 \%)$ \\
\hline$>24$ to 30 months & $18 / 75(24.0 \%)$ \\
\hline$>30$ to 36 months & $11 / 75(14.7 \%)$ \\
\hline$>36$ months & $10 / 75(13.3 \%)$ \\
\hline Therapy $\%$ receiving chemotherapy/hormone therapy) & $44 / 75(58.7 \%)$ \\
\hline Surgical Procedure & \multicolumn{2}{|l|}{} \\
\hline Lumpectomy & $27 / 73(37.0 \%)$ \\
\hline Mastectomy & $46 / 73(63.0 \%)$ \\
\hline Breast reconstruction & $35 / 46(76.1 \%)$ \\
\hline & \\
\hline
\end{tabular}

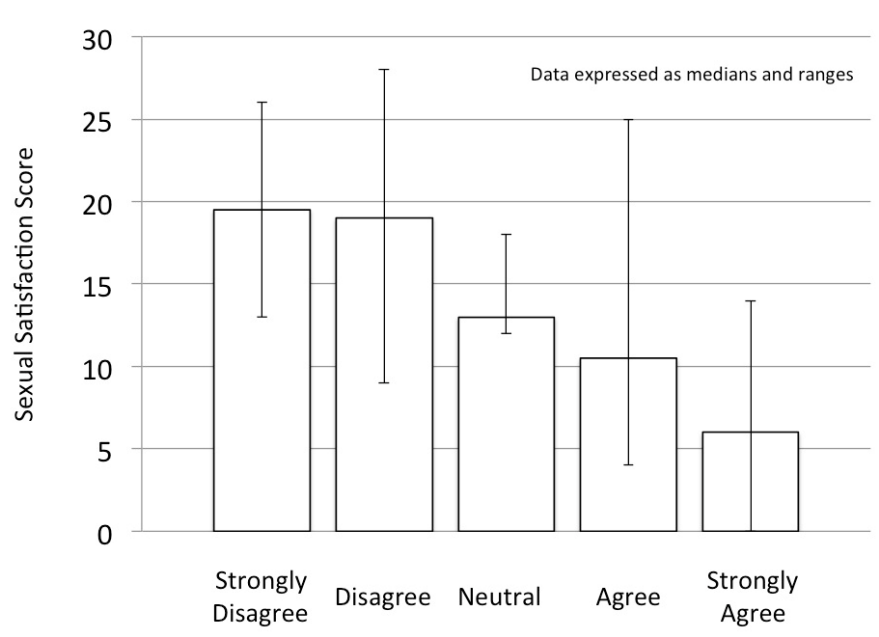

Figure 1. Relationship between satisfaction score and response to the statement "I feel that my breast cancer treatment negatively impacted my intimate relationship". 
Table 2. Physical factors cited by responders as interfering with sexual function.

\begin{tabular}{|c|c|c|c|c|c|c|}
\hline & Not noted ${ }^{*}$ & Not at all & A little bit & Somewhat & Quite a bit & Very much \\
\hline Fatigue & 6 & $13 / 55(23.6 \%)$ & $14 / 55(25.5 \%)$ & $10 / 55(18.2 \%)$ & $12 / 55(21.8 \%)$ & $6 / 55(10.9 \%)$ \\
\hline Pain & 18 & $14 / 43(32.6 \%)$ & $10 / 43(23.3 \%)$ & $11 / 43(25.6 \%)$ & $5 / 43(11.6 \%)$ & $3 / 43(7.0 \%)$ \\
\hline Weight loss & 45 & $12 / 16(75.0 \%)$ & $1 / 16(6.3 \%)$ & $1 / 16(6.3 \%)$ & $2 / 16(12.5 \%)$ & $0 / 16(0.0 \%)$ \\
\hline Weight gain & 36 & $8 / 25(32.0 \%)$ & $8 / 25(32.0 \%)$ & $4 / 25(16.0 \%)$ & $4 / 25(16.0 \%)$ & $1 / 36(4.0 \%)$ \\
\hline Hair loss & 55 & $4 / 6(66.7 \%)$ & $0 / 6(0.0 \%)$ & $0 / 6(0.0 \%)$ & $1 / 6(16.7 \%)$ & $1 / 6(16.7 \%)$ \\
\hline Medical device & 55 & $4 / 6(66.7 \%)$ & $2 / 6(33.3 \%)$ & $0 / 6(0.0 \%)$ & $0 / 6(0.0 \%)$ & $0 / 6(0.0 \%)$ \\
\hline Scars & 1 & $26 / 60(43.3 \%)$ & $14 / 60(23.3 \%)$ & $12 / 60(20.0 \%)$ & $8 / 60(13.3 \%)$ & $0 / 60(0.0 \%)$ \\
\hline Breast tenderness & 21 & $10 / 40(25.0 \%)$ & $16 / 40(40.0 \%)$ & $8 / 40(20.0 \%)$ & $6 / 40(15.0 \%)$ & $0 / 40(0.0 \%)$ \\
\hline Hot flashes & 17 & $11 / 44(25.0 \%)$ & $15 / 44(34.1 \%)$ & $10 / 44(22.7 \%)$ & $5 / 44(11.4 \%)$ & $3 / 44(6.8 \%)$ \\
\hline
\end{tabular}

* Physical factor not present, or not noted within the past 30 days.

$70 \%$ of the 46 patient in the mastectomy group perceived a negative impact on their sexual functioning $(\mathrm{p}=0.07)$. We further analyzed the mastectomy group into those who underwent reconstruction versus those without reconstruction finding that those who underwent reconstruction perceived an increased negative impact on their sexual function compare to those that did not have reconstruction. However, this was not found to be statistically significant $(\mathrm{p}=.21)$.

\section{Discussion}

The 2005 IOM report, "From Cancer Patient to Cancer Survivor: Lost in Transition," emphasized that a lack of definitive guidance on what constitutes best practices in caring for survivors contributes to wide variation in care. Additionally, primary care physicians are often unfamiliar with the consequences of cancer and its treatment and seldom receive explicit survivor care guidance about potential treatment effects from oncologists [3]. Increased efforts are needed to raise awareness of the medical, functional, and psychosocial consequences of cancer and its treatment, to define what constitutes a quality health care plan for cancer survivors and identify strategies to implement it, and to improve the quality of life of cancer survivors through policies that provide access to necessary medical and psychosocial services.

Our data support a growing body of evidence that suggest sexual problems can be a significant side effect from breast cancer treatment. The majority of our population, $64 \%$, felt that their breast cancer treatment caused some level of intimate relationship dysfunction. Less than $25 \%$ of women who perceived dysfunction brought up these concerns to their physician; however, approximately $60 \%$ of women wanted this addressed by a physician initiated conversation. A perceived negative impact due to treatment was associated with less intimate relationship satisfaction overall. A correlation between the presence of scars, the type of surgical intervention, and feeling that treatment had a negative impact on intimate relationships was identified. These data support a growing body of evidence that suggests sexual problems can be a significant side effect from breast cancer treatment.

The PROMIS system has previously been validated in assessing sexual function in cancer patients. In 2012 Flynn noted that across all cancer types, sexual function and intimacy were significantly impacted but fatigue, treatment related hair loss, weight gain, and organ loss or scarring [11]. Our data support the negative effect of treatment changes, especially scarring, on sexual functioning.

A high prevalence of sexual dysfunction is breast cancer patient has previously been documented. Panjari et al. found that $70 \%$ of breast cancer survivors experience some form of sexual dysfunction [12]. Our data suggest that two-thirds of breast cancer patients experience some disturbance in intimacy or sexual functioning. Unfortunately, pre-treatment data on patients' sexual function is not available for comparison, thus we are unable to quantify the degree of change that treatment may have caused. However, our patient set attributes their changes to treatment.

While our data demonstrates that the majority of breast cancer patients are experiencing symptoms, only a minority, $25 \%$, volunteer this information to their health care provider. However, $60 \%$ of patients want their provider to initiate a conversation about sexual functioning and intimacy. The reasoning behind this preference (embarrassment, insecurity, etc.) was not addressed by our survey. Flynn et al. published similar results with $74 \%$ of their patients feeling that discussion about sexual problems with their health care professionals was important. However this issue was addressed much less frequently, and varied by cancer type (29\% breast, $23 \%$ lung, 39\% colorectal, and 79\% prostate) [11].

Even when providers are addressing sexuality, they may often not be addressing patients' concerns. Hordern and Street demonstrated a significant mismatch in physician versus patient expectations. Their data suggest that most patients desire information and practical strategies to cope with changes in sexuality and intimacy [13]. In contrast, most professionals focused on sexuality related to fertility, contraception, menopausal status, or erectile function. They also showed many stereotypical assumptions of providers of patients' sexuality based on age, gender, diagnosis, culture, and partnership status.

Our data highlight that healthcare professionals are not identifying quality of life concerns in breast cancer survivors. Multiple authors have advocated for the use of standardized quality of life assessments. Standardized assessments have been shown to improve communication between patients and providers. Patients who participate in quality of life assessments have also been shown to receive significantly more counseling than those without assessments [14].

Finally, an interesting finding in this study is the increased incidence of perceived negative impact on sexual function in mastectomy patients compared with breast conservation patients. And, while not statistically significant, reconstruction appeared to have an additional negative impact on sexual function. This finding may be due to a variety of reasons and cofounding variables such as stage of disease, additional treatment needed, additional required surgery, or greater physical discomfort. The incidence of pain and/or discomfort after a mastectomy with reconstruction has been reported as high as 70\% [15]. While the data regarding body image and sexual dysfunction in women with and without reconstruction is mixed, most suggest an improved overall body image and intimacy in women undergoing successful reconstruction [15]. However, as we continue to see a growing trend of increasing numbers of mastectomies, this information is important to consider as one of the many factors in a patients' choice of surgery.

Limitations of this study include the low number of participants with an $18 \%(n=75)$ of responders completing both the informed 
consent and the survey. The use of a survey as a means to acquire data is also associated with responder bias, those experiencing sexual dysfunction being more likely to complete and return the survey. Finally, approximately two-thirds of survey responders had mastectomies which are in contrast to the actual clinic population characteristics in which the majority of patients undergo breast conservation surgery indicating possible responder bias.

Future research is needed in how to better assess both the presence and the origin of patients' sexual dysfunction in a clinical setting. Focused effort should be made to include sexual dysfunction assessment in routine breast cancer survivorship visits. Better definition of the underlying issues of a patient's dysfunction whether they be psychological, anatomical, or relationship based, will enable the healthcare team to provide personalized care and support. Focusing on patients desire to obtain information and practical strategies to cope with changes in sexuality and intimacy should be a primary objective of the healthcare provider inquiry.

\section{References}

1. Jemal A, Siegel R, Xu J, Ward E (2010) Cancer statistics, 2010. CA Cancer J Clin 60: 277-300. [Crossref]

2. Institute of Medicine and National Research Council (2005) From Cancer Patient to Cancer Survivor: Lost in Transition. Washington, DC: The National Academies Press.

3. Hulbert-Williams N, Neal R, Morrison V, et al. (2011) Anxiety, depression and quality of life after cancer diagnosis: what psychosocial variable best predict how patients adjust? Psychooncology 21: 857-867. [Crossref]

4. Harrington CB, Hansen JA, Moskowitz M, Todd BL, Feuerstein M (2010) It's not over when it's over: long-term symptoms in cancer survivors--a systematic review. Int $J$ Psychiatry Med 40: 163-181. [Crossref]

5. Fallowfield LJ (1995) Assessment of quality of life in breast cancer. Acta Oncol 34: 689-694. [Crossref]
6. Knobf MT (2011) Clinical update: psychosocial response in breast cancer survivors. Semin Oncol Nurs 27: e1-e14. [Crossref]

7. Edwards PJ, Roberts I, Clarke J, et al. (2009) Methods to increase response to postal and electronic questionnaires. Cochrane Database Syst Rev 8: MR000008. [Crossref]

8. Dillman D (1978) Mail and telephone surveys. New York: Wiley.

9. Flynn KE, Jeffery DD, Keefe FJ, Porter LS, Shelby RA, et al. (2011) Sexua functioning along the cancer continuum: focus group results from the Patient-Reported Outcomes Measurement Information System (PROMISŽ). Psychooncology 20: 378386. [Crossref]

10. Cella D, Riley W, Stone A, et al. (2010) The Patient-Reported Outcomes Measurement Information System (PROMISŽ) developed and tested its first wave of adult selfreported health outcome item banks: 2005-2008. J Clin Epidemiol 63: 1179-1194. [Crossref]

11. Flynn KE, Reese JB, Jeffrey DD, Abernethy AP, Lin L, et al. (2012) Patient experiences with communication about sex during and after treatment for cancer. Psychooncology 21: 594-601. [Crossref]

12. Panjari M, Bell RJ, Davis SR (2011) Sexual function after breast cancer. $J$ Sex Med 8 : 294-302. [Crossref]

13. Hordern AJ, Street AF (2007) Communicating about patient sexuality and intimacy after cancer: mismatched expectations and unmet needs. Med J Aust 186: 224-227. [Crossref]

14. Detmar SB, Muller MJ, Schornagel NH, Wever LD, Aaronson NK (2002) Healthrelated quality-of-life assessments and patient-physician communication: a randomized controlled trial. JAMA 288: 3027-3034. [Crossref]

15. Gahm J, Wickman M, Brandberg Y (2010) Bilateral prophylactic mastectomy in women with inherited risk of breast cancer- prevalence of pain and discomfort, impact on sexuality, quality of life and feelings of regret two years after surgery. Breast 19: 462-469. [Crossref]

16. Eltahir Y, Werners LL, Dreise MM, van Emmichoven IA, Jansen L, et al. (2013) Quality-of-life outcomes between mastectomy alone and breast reconstruction: comparison of patient-reported BREAST-Q and other health-related quality-of-life measures. Plast Reconstr Surg 132: 201e-209e. [Crossref]

Copyright: (C2018 Zomerlei TA. This is an open-access article distributed under the terms of the Creative Commons Attribution License, which permits unrestricted use, distribution, and reproduction in any medium, provided the original author and source are credited. 